\title{
Efecto de la cooperación motriz en la vivencia emocional positiva: perspectiva de género
}

\author{
Pere Lavega* \\ Francisco Lagardera** \\ Jaume March*** \\ Gloria Rovira**** \\ Paulo Coelho Araújo*****
}

\begin{abstract}
Resumen: Se investigó, desde la perspectiva de género, la vivencia emocional suscitada con la práctica de situaciones motrices cooperativas. Participaron 309 estudiantes de 4 universidades españolas. Se empleó la escala validada de juegos y emociones (GES). Tras cada tarea, los alumnos anotaron la intensidad experimentada en las trece emociones consideradas y comentaron brevemente las causas que originaron la emoción más intensa. Se emplearon métodos mixtos al complementar el análisis de los datos cuantitativos con el estudio de comentarios cualitativos. Se confirmó la aportación de las situaciones motrices de juegos tradicionales, expresión e introyección para promover experiencias emocionales positivas en mujeres y hombres.
\end{abstract}

Palabras Clave: Educación física. Inteligencia emocional. Identidad de Género. Relaciones interpersonales.

/s/

\footnotetext{
*Departamento Fundamentos Motricidad y su Enseñanza. Instituto Nacional de Educación Física de Cataluña (INEFC). Universidad de Lleida. Lleida, Espanha. E-mail: plavega@inefc.es **Departamento Fundamentos Motricidad y su Enseñanza. Instituto Nacional de Educación Física de Cataluña (INEFC). Universidad de Lleida. Lleida, Espanha. E-mail: flagardera@inefc. es

${ }^{* * *}$ Facultad de Pedagogía y Psicología., Universidad de Lleida. Lleida, Espanha. E-mail: jmarch@pip.udl.cat

****Escuela Universitaria de la Salud y el Deporte. Universidad de Girona. Girona, Espanha. E-mail: gloria.rovira@cadscrits.edu.udg

*****Faculdade de Ciências do Desporto e Educação Física. Universidade de Coimbra. Coimbra, Portugal. E-mail: pcoelho@fcdef.uc.pt
} 


\section{INTRODUCCIÓN}

La Educación Física (EF) es una disciplina pedagógica que se sirve de situaciones motrices para lograr objetivos y competencias educativas en el alumnado. Ante la extraordinaria variedad de situaciones a elegir, uno de los principales retos de la EF es examinar la relación existente entre las vivencias motrices y los efectos que éstas originan en el alumnado (LAGARDERA; LAVEGA, 2003; MCCAUGHTRY, 2004; PARLEBAS, 2001). Desde este punto de vista la educación (física) actual puede jugar un papel destacado en cultivar el bienestar social y emocional del alumnado (BISQUERRA, 2003; ENGESER; RHEINBERG, 2008; FREDRICKSON, 1998; KELLER; BLESS, 2008; LAGARDERA, 2007; PEKRUN et al., 2009). El desafío educativo está servido si se desea desvelar los efectos que cualquier situación motriz tiene para proporcionar experiencias de bienestar, en este sentido, es preciso examinar dos realidades interconectadas: a) las propiedades que tiene toda situación motriz en tanto que sistema social que pre orienta las relaciones y también las emociones de los participantes; este artículo hace referencia exclusivamente a las tareas motrices de cooperación; y b) el actor (alumnado) con la valoración y significación afectiva que hace de cada situación motriz a partir de sus atributos personales (este artículo aborda las variables subjetivas de género - masculino y femenino -, tipo de emociones - se consideran trece -, que sienten los protagonistas al cooperar) y si posee o no historial deportivo.

\subsection{LA COOPERACIÓN, UN SISTEMA SOCIAL BASADO EN LA CONVIVENCIA}

Al cooperar las relaciones sociales se orientan hacia la ayuda recíproca con los demás, tal y como muestra claramente la praxiología motriz (PARLEBAS, 2001), disciplina científica que estudia las propiedades estructurales (lógica interna) de cualquier práctica motriz. En tanto que sistema social cualquier situación motriz cooperativa dispone de un orden estructural o lógica

Movimento, Porto Alegre, v. 20, n. 2, p. 593-618, abr.jun. de 2014. 
interna (MARIN, et al., 2012, ETXEBESTE, 2012, MATEU, 2010), entendiendo así al conjunto de sus rasgos pertinentes que la diferencian del resto de prácticas motrices (PARLEBAS, 2001) que pre orienta a los protagonistas a relacionarse con otros participantes, a utilizar el espacio, a emplear los objetos y de ajustarse a exigencias temporales. Saltar a la comba en equipo, representar una danza simbólica colectiva o ayudar a un compañero a explorar la escucha activa de su respiración, son tres ejemplos de prácticas motrices cooperativas que activan relaciones de ayuda mutua entre los participantes. Estamos ante el dominio de acción motriz de cooperación (ETXEBESTE, 2012; LAGARDERA; LAVEGA, 2003; PARLEBAS, 2001).

Este estudio dirige la atención hacia tres tipos de situaciones motrices cooperativas sin competición: juegos tradicionales, expresión motriz e introyección motriz (conciencia sensitiva), en general poco presentes en los programas de EF (LAGARDERA; LAVEGA, 2003).

\subsection{EduCAR DESDE EL BIENESTAR SOCIAL Y EMOCIONAL}

Una experiencia motriz está orientada hacia el bienestar si es capaz de desencadenar entre los participantes relaciones y emociones positivas, es decir, vivencias agradables en los protagonistas (FREDRICKSON 1998; RYAN; DECI, 2001) que les permita fluir y vivir experiencias óptimas (CSIKSZENTMIHALYI; MASSIMINI, 1985). Conviene a cualquier educador saber que un buen uso de las tareas motrices puede ser una fuente extraordinaria de aprendizajes asociados a fluir mediante la vivencia de emociones positivas (ENGESER; REINBERG, 2008; RYAN; DECI, 2001). Las emociones son un proceso multidimensional que afecta a diferentes niveles del organismo (fisiológico, cognitivo, motivacional, social) que en el contexto de la EF se manifiestan como respuesta ante las expectativas que generan las tareas motrices (PEKRUN et al., 2009). Cuando el alumno cubre esas expectativas se desencadenan emociones positivas tales como alegría, humor,

Movimento, Porto Alegre, v. 20, n. 2, p. 593-618, abr./jun. de 2014. 
amor y felicidad. Si la tarea motriz propuesta suscita malestar es porque se han activado emociones negativas como ira, ansiedad, miedo, tristeza, rechazo y vergüenza. También es posible observar emociones ambiguas como la sorpresa, esperanza y compasión que podrán tener una orientación positiva o negativa según las circunstancias (BISQUERRA, 2003; LAZARUS, 1991).

\subsection{GÉNERO Y COOPERACIÓN}

Tener en cuenta la perspectiva de género implica considerar los rasgos sociales y culturales de los protagonistas, por ejemplo, cuando se habla de liderazgo, agresividad o machismo como rasgo del género masculino, o de sensibilidad y dulzura como rasgo en el caso del género femenino. En cambio el término sexo se refiere a los rasgos biológicos que diferencian a los hombres de las mujeres (CHALABAEV, et al., 2013).

Estudios sobre los juegos tradicionales infantiles en España (ETXEBESTE, 2012) o de los deportes de adultos en Europa (LAVEGA, 2009), muestran que la cooperación es una de las interacciones sociales preferidas por el género femenino. En cambio los hombres casi ignoran la cooperación, dado que prefieren juegos que tienen una estructura de duelo entre individuos o entre equipos. El significado cultural y la adherencia a estereotipos culturales determinan que los chicos respondan con entusiasmo ante situaciones competitivas y que las chicas muestren dudas al respecto (CONTI; COLLINS; PICARIELLO, 2001; JOHNSON; ENGELHARD, 1992; KNIGHT; CHAO, 1989).

En el ámbito de la EF, el deporte ha sido históricamente un laboratorio de masculinidad (MCKAY; MESSNER; SABO, 2000), en cambio, otro tipo de prácticas motrices mucho más conectadas al significado cultural del género femenino como la expresión motriz, los juegos cooperativos, las gimnasias suaves o la introyección motriz (por ejemplo, el ajuste de la postura, los estiramientos de las cadenas musculares posteriores o la respiración consciente) siguen teniendo un papel marginal. De ahí la necesidad de avanzar en propuestas

Movimento, Porto Alegre, v. 20, n. 2, p. 593-618, abr.jun. de 2014. 
alternativas hacia una EF moderna y, plural que se plantee retos necesarios actualmente como educar el bienestar socioemocional.

A partir de esta referencia teórica la investigación plantea dos objetivos principales: a) analizar desde la perspectiva de género la intensidad de las emociones experimentadas en la práctica de situaciones motrices cooperativas basadas en juegos tradicionales, expresión e introyección motriz; y b) examinar desde la perspectiva de género el contenido de las argumentaciones referidas a las vivencias emocionales más intensas suscitadas con su práctica.

\subsection{EL HISTORIAL DEPORTIVO}

El historial deportivo corresponde a las experiencias deportivas previas continuadas en el tiempo que tienen algunas personas (CASTEJÓN, 2003). Según se disponga o no de estos antecedentes deportivos las personas pueden presentar diferencias en el uso inteligente de sus emociones y en sus estados de ánimo (ANDRADE; ARCE; ARMENTAL; DE FRANCISCO, 2011), así como mantener actitudes diferentes ante la competición deportiva.

\section{Método}

\subsection{PARTICIPANTES}

Se estudiaron 309 alumnos (71 chicas y 338 chicos; rango de edad de 19-22 años; Medad = 19.6 años, DT = 2.32) de cuatro universidades españolas: Barcelona (80 estudiantes: 21 chicas y 59 chicos), Girona (86 estudiantes: 17 chicas y 69 chicos), Lleida (94 estudiantes: 19 chicas y 75 chicos) y Zaragoza en sus sedes de Huesca (52 estudiantes: 14 chicas y 38 chicos) y de Zaragoza (14 chicas). Los participantes estudiaban primer curso de ciencias de la actividad física y el deporte o en la diplomatura de maestro en EF. Todos ellos participaron voluntariamente. Este estudio fue aprobado por el comité de ética de la universidad de Lleida.

Movimento, Porto Alegre, v. 20, n. 2, p. 593-618, abr.jun. de 2014. 
2.2 INSTRUMENTOS Y PROCEDIMIENTOS

Se realizaron 2 sesiones de 1,5 horas de situaciones motrices cooperativas de juegos tradicionales, expresión motriz y tareas de introyección motriz o de atención exploración del propio cuerpo tanto interna como exteroceptiva. En todos los casos los docentes de la experiencia se limitaron a describir la situación motriz y acto seguido pedir al alumnado que procediera a su práctica. Tan sólo intervenían en caso de que hubiera alguna duda sobre la tarea a realizar.

Identificación de la intensidad en la vivencia de emociones y explicación subjetiva de la intensidad emocional. Se empleó la escala GES (Games and Emotion Scale) validada por Lavega, March y Filella (2013) para identificar la intensidad emocional experimentada en cada tarea motriz. Las principales propiedades psicométricas de esa escala fueron: aceptabilidad: los valores de asimetría $(<2.0)$ y curtosis $(<7.0)$ cumplieron los criterios de normalidad. Fiabilidad: coeficiente alpha de Cronbach $(\mathrm{n}=851 ; \alpha=.92)$. Se obtuvieron valores similares para cada tipo de emoción: positiva $(\alpha=.92)$, negativa $(\alpha=.88) \mathrm{y}$ ambigua $(\alpha=.93)$. El análisis factorial confirmatorio reprodujo de manera adecuada la estructura de la escala y mostró índices aceptables $(\mathrm{CMIN} / \mathrm{g} .1 .=7.014 ; \mathrm{NFI}=.813 ; \mathrm{IFI}=.836 ; \mathrm{CFI}=.833 ; \mathrm{RMSEA}=$ $.08 ; \mathrm{LO} 90=.072-\mathrm{HI} 90=.09$ ).

Al finalizar cada actividad, los alumnos anotaron en su cuestionario personal el nivel de intensidad (de 0 a 10) que habían experimentado en trece emociones básicas (BISQUERRA, 2003). Cero significaba que no habían sentido esa emoción y diez que se había vivido con máxima intensidad. Acto seguido el alumno escribía un breve comentario para explicar la emoción más intensa experimentada (en caso de empate entre emociones podían comentar un máximo de tres emociones).

\subsection{ANÁLISIS DE LOS DATOS}

Se empleó la combinación de métodos (BERICAT, 1998), para fortalecer la validez de los hallazgos obtenidos a partir del

Movimento, Porto Alegre, v. 20, n. 2, p. 593-618, abr./jun. de 2014. 
análisis estadístico de los datos cuantitativos mediante el análisis de contenido de los comentarios de las vivencias emocionales más intensas.

Análisis de los datos cuantitativos (grado de intensidad de las emociones vivenciadas). La prueba de Kolmogorov-Smirnov, mostró que los datos eran no paramétricos, ya que seguían una distribución muy asimétrica de las intensidades. Por este motivo se siguió un modelo basado en ecuaciones de estimación generalizadas (GEE) para considerar la correlación entre las puntuaciones del mismo sujeto. Se utilizaron distribuciones de la familia Gausiana, con una estructura de correlación intercambiable. Se aplicaron comparaciones múltiples post-hoc en todos los factores de más de dos categorías. Se empleó el software estadístico SPSS v.19.0. El modelo consideró el Tipo de emoción (positiva, negativa y ambigua) como factor intra-sujetos. Los factores entre-sujetos fueron: 1) Tipo de práctica (juegos, expresión e introyección), 2) Género (masculino, femenino), y 3) Historial Físico-deportivo (con historial, sin antecedentes).

Análisis de los datos cualitativos (explicaciones subjetivas de la intensidad emocional). Este apartado corresponde a los comentarios que hacían los alumnos sobre las emociones que habían alcanzado los valores más intensos en cada juego.

Proceso de codificación. Un equipo de seis personas que imparten fundamentos de Praxiología Motriz en asignaturas de grado o máster universitario (dos en expresión motriz, dos en juegos deportivos y dos en introyección motriz) elaboró un manual de instrucciones que incluyó criterios y reglas a seguir que asegurasen la exclusividad, la clarificación y delimitación de las diez categorías. Se aplicó un análisis de contenido a las explicaciones de las emociones más intensas en función de si los comentarios se referían a los rasgos estructurales de las prácticas motrices (lógica interna) o si correspondían al contexto de esas prácticas (lógica externa). Se codificaron cinco categorías concernientes a la lógica interna: a) relación interna (tipo de relación motriz producida entre

Movimento, Porto Alegre, v. 20, n. 2, p. 593-618, abr.jun. de 2014. 
participantes) "me ha gustado mucho cooperar con mis compañeros", "me producía placer intervenir con la ayuda de mi compañera"; b) espacio interno (uso del terreno de juego) "me ha costado desplazarme de un lado a otro sin desequilibrarme", "me ha gustado moverme de un sitio a otro mientras saltaba"; c) tiempo interno, de acción, muy especialmente hechos y acontecimientos ocurridos en el transcurso de las prácticas (aspectos referidos a momentos del juego) "he podido disfrutar de cada instante", "el tiempo de juego era muy breve"; d) material interno, referido al uso del material para participar (maneras de usar los objetos) "me costaba controlar la pelota", "el balón botaba demasiado"; y e) reglas (alusión a aspectos generales sobre condiciones o características de la práctica motriz) "la tarea no ha sido difícil", "este juego ha sido divertido".

Las cinco categorías pertenecientes a la lógica externa del juego fueron: a) tiempo externo (características del tiempo p.ej. horario y meteorología), "hacía mucho frío en la sala", "era la última hora de la semana y se notaba"; b) espacio externo (las condiciones de mantenimiento del terreno de juego), "el suelo estaba sucio", "el pavimento era muy resbaladizo"; c) material externo (componentes básicos del material de juego), "las pelotas estaban hechas de papel de periódico", "el papel de periódico se rompía con facilidad"; d) relación externa (referida a atributos permanentes de los jugadores), "los chicos eran menos flexibles y les dolía la espalda al estirarse"; "en mi equipo estaban los más fuertes"; y e) estados transitorios (referido a las alusiones a rasgos y circunstancias transitorias de las personas), "no he parado de reír", "hoy tenía mucho sueño".

Esos textos se clasificaron en función de presencia (1) o ausencia (0) de términos asociados a rasgos de la lógica interna o externa. La unidad de codificación que se eligió fue el texto completo de cada comentario. Por ello algunos mensajes podían ser codificados con más de una categoría asociada a la lógica interna y a la lógica externa de las prácticas motrices realizadas. A continuación mostramos algunos ejemplos en los que se identifican más de una variable:

Movimento, Porto Alegre, v. 20, n. 2, p. 593-618, abr.jun. de 2014. 
Comentarios referidos únicamente a la lógica interna: "La ayuda de mis compañeros (relación interna) ha sido fenomenal para poder desplazarnos de un sitio a otro (espacio interno); "al principio del juego (tiempo interno) nos ha costado mover el paracaídas (material interno)"

Comentarios referidos únicamente a la lógica externa: "He intervenido con mi mejor amiga (relación externa) y no hemos parado de reírnos (estados transitorios)"; "Me lo he pasado muy bien (estados transitorios) ya que me recordaba a un juego de mi infancia (tiempo externo)".

Comentarios referidos a la lógica interna y a la lógica externa: "Cuando hemos terminado el juego (tiempo interno) no podíamos parar de reír (estados transitorios)"; "Los chicos (relación externa) empleaban el balón de manera muy original (material interno)"

Cálculo de fiabilidad entre codificadores. El equipo de investigadores se distribuyó por parejas para analizar los comentarios de cada tipo de práctica motriz (juegos, expresión e introyección) y procedió a codificar cada uno de los tres grupos de textos de manera independiente. Cada pareja de codificadores participó de modo independiente en un proceso de entrenamiento de unas 40 horas sobre los comentarios del tipo de tarea motriz que debían analizar. En ese proceso se estableció que el acuerdo entre codificadores se daría cuando las dos personas coincidieran en la identificación de la presencia de todas las variables sobre un mismo comentario. Durante ese proceso se analizaron 80 comentarios, durante 10 sesiones y se identificaron las coincidencias y posibles desacuerdos. Las dudas surgidas en tres grupos de parejas se discutieron y resolvieron entre los seis investigadores. Posteriormente para obtener el cálculo de la fiabilidad intercodificadores se seleccionaron al azar 100 comentarios (de cada tipo de práctica motriz) que fueron analizados individualmente. La concordancia entre los observadores para cada práctica motriz se calculó mediante el coeficiente kappa de Cohen (COHEN, 1960), obteniendo los siguientes valores: 0.86 en los juegos tradicionales, 0.84 en la expresión motriz y 0.79 en la introyección motriz.

Movimento, Porto Alegre, v. 20, n. 2, p. 593-618, abr.jun. de 2014. 
Análisis de los datos cualitativos mediante el árbol de clasificación CHAID. Se empleó el método de crecimiento del árbol CHAID (detector de interacción automática de Chicuadrado basados en el modelo Answer-Tree C SPSS Árboles de Clasificación TM 13.0.). Se aplicó un sistema de validación cruzada, siendo 50 el número mínimo de sujetos en los nodos terminales y 3 niveles máximos de árbol.

Se generó un árbol de clasificación CHAID a partir del criterio de presencia o ausencia de explicaciones para cada variable de la lógica interna y de la lógica externa. Además se elaboró un árbol general que diferenció los comentarios que se dirigían a la lógica interna, a la lógica externa o a ambas variables. Se consideraron 4 variables predictivas: 1) tipo de emoción (positiva, negativa y ambigua); 2) género (femenino y masculino); 3 ) tipo de práctica (juego, expresión, introyección); e 4) historial deportivo (presencia y ausencia de antecedentes deportivos).

\section{Resultados}

\subsection{INTENSIDAD EMOCIONAL. RESULTADOS A PARTIR DE LOS DATOS CUANTITATIVOS}

El análisis estadístico de los datos cuantitativos $(\mathrm{N}=52741)$, mediante GEE, reflejó diferencias significativas entre los tres tipos de emociones $(p<.001)$. Las emociones positivas obtuvieron los valores más intensos $(\mathrm{M}=4.00)$, las emociones negativas activaron valores significativamente más bajos $(\mathrm{M}=0.39, \mathrm{p}<.001)$ y las emociones ambiguas registraron valores significativamente inferiores a las emociones positivas $(\mathrm{M}=1.51, \mathrm{p}<.001)$.

No se observaron diferencias significativas entre ambos géneros $(p=.683)$. Sin embargo, la interacción significativa entre género y tipo de emoción $(\mathrm{p}=.018)$ mostró que los hombres vivieron las emociones negativas con mayor intensidad $(\mathrm{M}=0.45)$ que la mujeres $(\mathrm{M}=0.33, \mathrm{p}=.040)$, y que las mujeres experimentaron las

Movimento, Porto Alegre, v. 20, n. 2, p. 593-618, abr.jun. de 2014. 
Efecto de la cooperación motriz en la vivencia...

emociones positivas más intensamente $(\mathrm{M}=4.19)$ que los hombres $(\mathrm{M}=3.82, \mathrm{p}<.001)$.

Tabla 1. Efectos del modelo de ecuaciones de estimación generalizadas

\begin{tabular}{cccc}
\hline & Chi-cuadrado de Wald & gl & Sig. \\
\hline Tipo emoción (Emo_3) & 765,322 & 2 & $<.001$ \\
Género & 0,166 & 1 & .683 \\
Tipo práctica & 127,466 & 2 & $<.001$ \\
Historial deportivo & 0,363 & 1 & .547 \\
Emo_3* género & 8,059 & 2 & .018 \\
Emo_3* Tipo práctica & 154,213 & 4 & $<.001$ \\
Emo_3* Historial Deportivo & 1,786 & 2 & .409 \\
Género * Tipo práctica & 8,971 & 2 & .011 \\
Género * Historial Deportivo & 1,269 & 1 & .260 \\
Practica * Historial Deportivo & 9,140 & 2 & .010 \\
\hline
\end{tabular}

Nota: En fondo oscuro se destaca el comportamiento del factor género.

Fuente: Datos de los autores y autora

Figura 1. Comportamiento de la interacción género y tipo de emoción

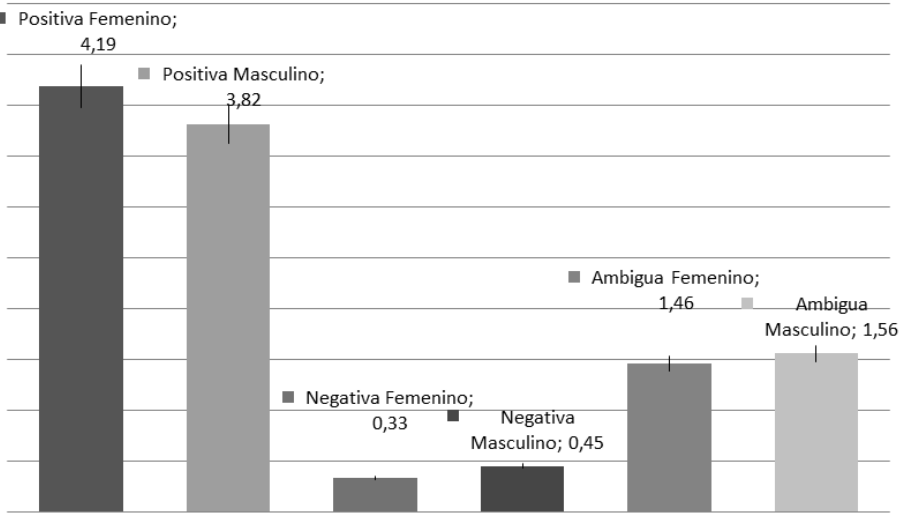

Fuente: Datos de los autores y autora

Movimento, Porto Alegre, v. 20, n. 2, p. 593-618, abr.jun. de 2014. 
Se observaron diferencias significativas en las intensidades emocionales registradas en los tres tipos de práctica $(p<.001)$. La introyección motriz provocó valores de intensidad emocional más bajos que los juegos y la expresión $(\mathrm{p}<.001)$. No se observaron diferencias significativas entre juegos $(\mathrm{M}=2.32)$ y expresión $(\mathrm{M}$ $=2.35, \mathrm{p}=.696)$.

La interacción entre tipo de práctica y tipo de emoción fue significativa $(\mathrm{p}<.001)$. La expresión originó emociones negativas más intensas $(\mathrm{M}=0.6)$ que los juegos $(\mathrm{M}=0.39, \mathrm{p}=.008)$.

La interacción género y tipo de práctica fue significativa $(\mathrm{p}=.011)$. La diferencia de intensidad entre los valores altos de juegos o expresión y los valores inferiores en introyección, fue significativamente superior en los hombres $(\mathrm{p}<.001)$.

Figura 2. Comportamiento de la interacción género y tipo de práctica motriz

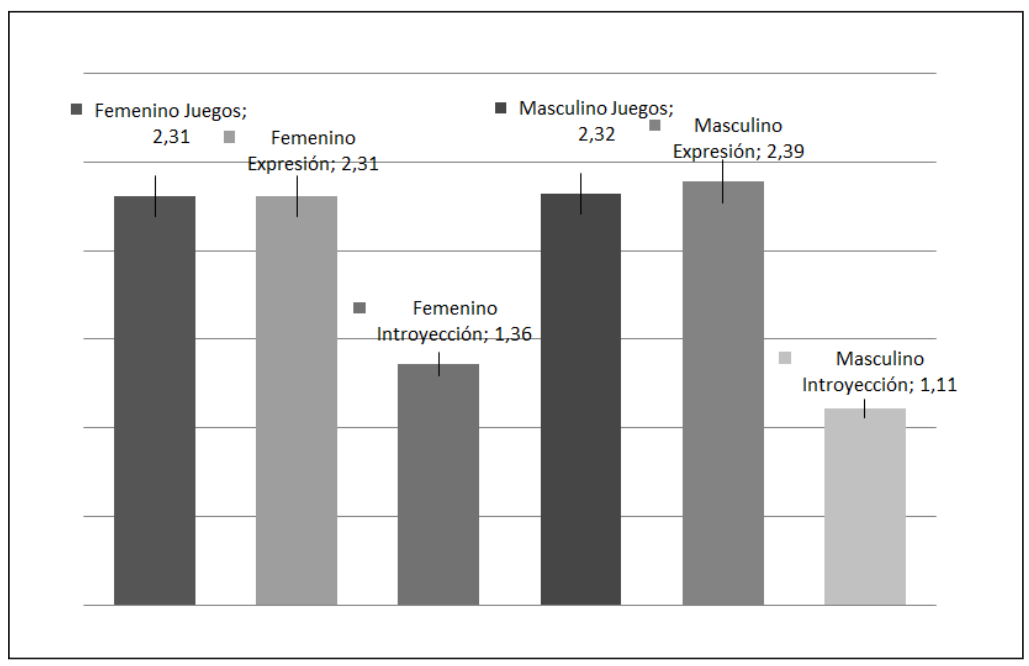

Fuente: Datos de los autores y autora

No se observaron diferencias significativas en el factor historial deportivo $(\mathrm{p}=.547)$. La interacción entre tipo de emoción

Movimento, Porto Alegre, v. 20, n. 2, p. 593-618, abr.jun. de 2014. 
$\mathrm{y}$ disponer de historial no fue significativa $(\mathrm{p}=.409)$. Tampoco existieron diferencias significativas en la interacción género y el historial deportivo $(\mathrm{p}=.260)$.

Se registraron diferencias significativas en la interacción tipo de práctica motriz y el historial deportivo $(\mathrm{p}=.010)$. Las prácticas motrices de introyección activaron valores significativamente más intensos en las personas que no tenían antecedentes deportivos $(\mathrm{M}=1.36)$ que en los participantes con historial deportivo $(\mathrm{M}=1.11, \mathrm{p}=.49)$. En cambio, no se encontraron diferencias significativas en los juegos ni en la expresión entre ambos tipos de participantes.

\subsection{EXPLICACIONES DE LAS EMOCIONES INTENSAS. RESULTADOS A} PARTIR DE LOS DATOS CUALITATIVOS

Los 943 comentarios recogidos originaron 11 árboles con nodos ordenados jerárquicamente en categorías homogéneas de relaciones entre las cuatro variables independientes (tipo emoción, género, tipo de práctica e historial deportivo) y la variable dependiente, existencia o no de comentarios para cada criterio de la lógica interna (reglas, tiempo interno, espacio interno, relación interna, objeto interno) y de la lógica externa (estados transitorios, tiempo externo, espacio externo, relación externa y objeto externo).

La técnica de segmentación jerárquica identificó que la emoción fue la principal variable predictiva de las interpretaciones en seis árboles (en cinco como variable del primer nivel de los árboles). El género fue la segunda variable predictiva en cinco árboles (dos como variable de primer nivel y tres como variable de segundo nivel). El historial fue la tercera variable predictiva en cinco árboles (tres como variable de primer nivel, una como variable de segundo nivel y una como variable del tercer nivel).

Movimento, Porto Alegre, v. 20, n. 2, p. 593-618, abr.jun. de 2014. 
Tabla 2. Distribución de los comentarios, nodos y variables predictivas en los diferentes árboles de clasificación

\begin{tabular}{cccccc}
\hline $\begin{array}{c}\text { Nombre del } \\
\text { Árbol }\end{array}$ & $\begin{array}{c}\% \\
\text { sentencias }\end{array}$ & $\begin{array}{c}\text { Núm. } \\
\text { Nodos }\end{array}$ & $\begin{array}{c}\text { Variables } \\
\text { predictivas } \\
\text { 1er. Nivel }\end{array}$ & $\begin{array}{c}\text { Variables } \\
\text { predictivas } \\
\mathbf{2}^{\mathbf{0}} \text { Nivel }\end{array}$ & $\begin{array}{c}\text { Variables } \\
\text { predictivas } \\
\text { 3er. Nivel }\end{array}$ \\
\hline $\begin{array}{c}\text { L. Interna-L. } \\
\text { Externa } \\
\text { Reglas }\end{array}$ & $100 \%$ & 4 & Género (2 nodos) & Historial (2 nodos) & - \\
Tiempo Interno & $22,1 \%$ & 6 & Historial (2 nodos) & $\begin{array}{c}\text { Emoción (2 nodos) } \\
\text { Género (2 nodos) }\end{array}$ & - \\
Espacio Interno & $35,6 \%$ & 6 & Emoción (3 nodos) & Género (2 nodos) & - \\
Relación Interna & $35,9 \%$ & 2 & Género (2 nodos) & - & - \\
Objeto Interno & $8,8 \%$ & 2 & Emoción (2 nodos) & - & - \\
Estados & $32,1 \%$ & 8 & Emoción (2 nodos) & Emoción (2 nodos) & $\begin{array}{c}\text { Historial } \\
(4 \text { nodos) }\end{array}$ \\
Transitorios & $4,5 \%$ & 0 & - & - & - \\
Tiempo Externo & $0,6 \%$ & 0 & - & - & - \\
Espacio Externo & $0,6 \%$ & - & - & - \\
Relación Externa & $4,1 \%$ & 0 & 2 & Emoción (2 nodos) & - \\
Objeto Externo & $1,4 \%$ & $2 \%$ & & - \\
\hline
\end{tabular}

Fuente: Datos de los autores y autora

Nota. En fondo oscuro se destaca la presencia de la variable género en cada uno de los árboles de clasificación.

\subsection{GÉNERO Y COMENTARIOS REFERIDOS A LA LÓGICA INTERNA Y/O LÓGICA EXTERNA}

El primer árbol de Lógica Interna-Externa integrado por todos los comentarios permitió identificar la tendencia general de las explicaciones de los protagonistas. La mayor parte de esas narraciones $(60.9 \%)$ aludieron a aspectos de la lógica interna de las tareas. El porcentaje inferior (4,2\%) correspondió a comentarios referidos a la lógica externa de las actividades realizadas. En un nivel intermedio se situaron las sentencias dirigidas simultáneamente a aspectos de la lógica interna y de la lógica externa de las tareas (34.9\%). El género fue la primera variable predictiva en el árbol de Lógica Interna-Externa. Se observaron diferencias significativas entre ambos géneros $(\mathrm{p}=.004$, Chi-cuadrado $=11.252, \mathrm{df}=2)$. La

Movimento, Porto Alegre, v. 20, n. 2, p. 593-618, abr./jun. de 2014. 
Efecto de la cooperación motriz en la vivencia...

variable género originó dos ramas (nodos 1 y 2). En un segundo nivel el Historial Deportivo fue la variable independiente que el árbol asoció a los comentarios de las mujeres, mientras que no se observó ninguna variable predictiva asociada a las explicaciones de los hombres. Se encontraron diferencias significativas entre las participantes con historial deportivo respecto a no tener antecedentes deportivos $(\mathrm{p}=.001$, Chi-cuadrado $=14.998, \mathrm{df}=2)$.

El análisis global del árbol identificó dos patrones de resultados extremos. Los porcentajes más elevados se asignaron a la lógica interna y al género masculino. Los valores inferiores correspondieron a la lógica externa y al género femenino con historial deportivo.

Figura 3. Árbol de clasificación referido las variables Lógica Interna y Lógica Externa

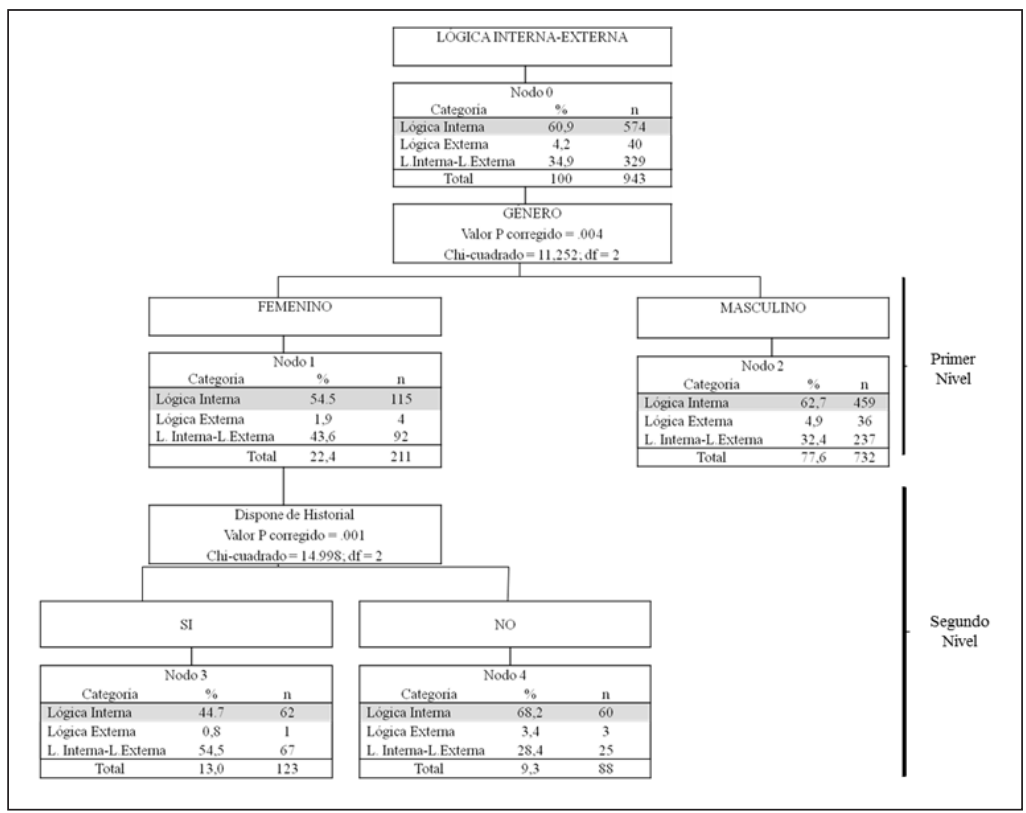

Fuente: Datos de los autores y autora

Movimento, Porto Alegre, v. 20, n. 2, p. 593-618, abr.jun. de 2014. 
Sólo cinco factores alcanzaron un nivel de presencia de comentarios superior al $20 \%$. Cuatro factores correspondieron a variables de la lógica interna de las prácticas motrices: Reglas (58 \%), Relación Interna (35.9\%), Espacio Interno (35.6 \%), y Tiempo Interno $(22.1 \%)$. La única variable externa que estuvo presente de modo representativo en los comentarios fue la de Estados Transitorios (32.1\%). En todos estos árboles, excepto en el árbol de Reglas, el género intervino como variable predictiva de los comentarios.

Género y comentarios referidos a la Relación Interna. El género fue la única variable predictiva. Se encontraron diferencias significativas entre géneros $(\mathrm{p}=.009$, Chi-cuadrado $=6.914 ; \mathrm{df}$ $=1$ ). Las mujeres originaron mayor porcentaje (43.6\%) que los hombres $(33.7 \%)$ de comentarios referidos al tipo de relación motriz que originaron las tareas cooperativas.

Género y comentarios referidos al Espacio Interno. El tipo de emoción fue la primera variable predictiva. No se encontraron más variables independientes para los comentarios asociados a emociones negativas y ambiguas. Sin embargo el género intervino como variable independiente asociada a las emociones positivas. Se observaron diferencias significativas entre géneros $(p=.045$, Chi-cuadrado $=4.02 ; \mathrm{df}=1$ ) en esas emociones. Las mujeres originaron mayor porcentaje $(34.1 \%)$ de comentarios sobre emociones positivas que los hombres (26\%).

Género y comentarios referidos al Tiempo Interno. El historial deportivo fue la primera variable predictiva. En los participantes sin historial el género intervino como variable explicativa en el segundo nivel del árbol. Se encontraron diferencias significativas entre géneros que no tenían antecedentes deportivos $(\mathrm{p}=.002$, Chicuadrado $=9.939 ; \mathrm{df}=1)$. Las mujeres originaron mayor presencia de comentarios $(25 \%)$ que los hombres $(8.4 \%)$.

Género y comentarios referidos a Estados Transitorios (Lógica Externa). Fue el único árbol de variables de lógica externa que incluyó al género como variable predictiva, junto a las variables emoción e historial deportivo. Este árbol originó 4 nodos. Se encontraron diferencias significativas entre las emociones

Movimento, Porto Alegre, v. 20, n. 2, p. 593-618, abr./jun. de 2014. 
positivas y negativas (nodo 1) y las emociones ambiguas (nodo 2), $(\mathrm{p}<.001$, Chi-cuadrado $=18.836, \mathrm{df}=1)$. El árbol se ramificó para el nodo emociones positivas-negativas a partir del género como siguiente variable predictiva. Existieron diferencias significativas entre ambos géneros $(\mathrm{p}=.001$, Chi-cuadrado $=10.359$, $\mathrm{df}=1)$. Las mujeres originaron mayor porcentaje de comentarios $(44.7 \%)$ que los hombres $(32.2 \%)$. La siguiente variable predictiva para ambos géneros fue el historial deportivo. Las mujeres con historial generaron mayor porcentaje de narraciones $(54.1 \%)$ que las que no tenían antecedentes deportivos (29.4\%). Esta superioridad se invirtió a favor de los hombres sin historial deportivo (44.3\%) respecto a los que tenían antecedentes deportivos (30.2\%).

\section{Discusıón}

Este trabajo investigó, desde la perspectiva de género, la relación entre diferentes tareas motrices cooperativas y el tipo de vivencia emocional, así como el historial deportivo, complementando el análisis cuantitativo con el cualitativo. El análisis de los datos cuantitativos (intensidad emocional) y cualitativos (narraciones que explican esa intensidad emocional) confirman el papel relevante de la cooperación en la promoción del bienestar social y emocional. Además se observan tres factores clave: a) la emoción (positiva, negativa y ambigua) que experimentan los participantes al cooperar, b) el género (femenino y masculino), y c) la presencia o ausencia de historial deportivo. La interacción de los factores atribuibles al sistema praxiológico (tipo de práctica motriz) y del protagonista de la acción o actor (emociones, género e historial deportivo) resulta clave para desvelar el bienestar asociado a esas vivencias emocionales.

\subsection{LA FUERZA DE LA LÓGICA INTERNA COOPERATIVA PARA SUSCITAR EMOCIONES POSITIVAS INTENSAS}

El dominio de acción motriz cooperativo origina valores intensos de emociones positivas, emociones negativas de baja intensidad y emociones ambiguas con valores intermedios. Estos

Movimento, Porto Alegre, v. 20, n. 2, p. 593-618, abr./jun. de 2014. 
hallazgos concuerdan con otros estudios sobre las prácticas motrices y las emociones (LAVEGA, et al., 2011; LAVEGA et al., 2013; TORRENTS et al., 2011).

El análisis de la intensidad emocional permite organizar las prácticas motrices cooperativas en dos grandes subdominios afectivos. El primer subdominio está constituido por el juego tradicional y la expresión motriz. En ambos casos, la atención de las acciones motrices se dirige hacia el mundo exterior, ya sea a través de una comunicación instrumental en los juegos cooperativos o de una comunicación referencial, orientada hacia una referencia externa y poética (las propias acciones son el mensaje) en la expresión motriz cooperativa (MATEU, 2010; PARLEBAS, 2001). En este subdominio se activan las emociones positivas y ambiguas más intensas. El otro subdominio lo constituye la introyección motriz, cuya lógica interna pre orienta a los participantes hacia la escucha activa y la exploración interna de sus conductas motrices. La interacción motriz cooperativa estimula $y$ favorece esta autoexploración.

No obstante, este tipo de vivencias activa emociones de menor intensidad que en los otros casos. En realidad, se trata de dos tipos de interacción cooperativa bien diferenciados. En el caso de los juegos tradicionales y la expresión motriz se comparte un objetivo común, por el que todos los participantes se ayudan en aras de la colectividad. Mientras que la cooperación en la introyección motriz ostenta un papel mucho más generoso, incluso podría decirse que solidario, ya que se coopera con el otro para ayudarle a sentirse mejor, para estimular su percepción de sí mismo y facilitarle el proceso de descubrimiento interno (LAGARDERA, 2007; ROVIRA, 2010).

El análisis de los datos cualitativos da como resultado que las variables de la lógica interna de las prácticas son las responsables de la vivencia intensa de las emociones positivas, dado que son las que aparecen mayoritariamente en sus justificaciones. La mayoría de las explicaciones corresponden a los árboles obtenidos a partir

Movimento, Porto Alegre, v. 20, n. 2, p. 593-618, abr.jun. de 2014. 
de las variables internas del sistema praxiológico: reglas, relación, tiempo y espacio. Sin embargo, los alumnos apenas realizaron comentarios referidos exclusivamente a motivos externos (lógica externa) de las prácticas motrices (4\% de los comentarios). Los datos han mostrado que los principales motivos que originan la vivencia emocional proceden de las características de las propias prácticas, más que otros temas de carácter externo a las situaciones motrices. Cada situación motriz introduce al alumno en el desafío de tener que solucionar problemas de relación motriz con otros participantes, con el espacio de juego, con el material y con el tiempo (ETXEBESTE, 2012; LAGARDERA; LAVEGA, 2003; PARLEBAS, 2001).

Es ese conjunto de relaciones internas el que desencadena vivencias emocionales intensas tal y como se ha observado en otros trabajos que ya han constatado que la lógica interna de los juegos deportivos (LAVEGA et al., 2013; LAVEGA et al., 2011), de la expresión motriz (TORRENTS et al., 2011) y la introyección motriz (LAGARDERA, 2007; ROVIRA, 2010) adquiere un papel destacado en la educación de la afectividad del alumnado. Este dato ya es en sí mismo un hallazgo de extraordinarias consecuencias pedagógicas: sería conveniente que el profesor de educación física identificara, haciendo uso de la Praxiología Motriz, la lógica interna cualquier práctica motriz que quiera emplear con los alumnos para diseñar programas de intervención en función de criterios rigurosos (MCCAUGHTRY, 2004) que sean congruentes con los objetivos pedagógicos planteados.

\subsection{GÉNERO Y VIVENCIA INTENSA DE EMOCIONES POSITIVAS}

Los datos cuantitativos muestran diferencias significativas entre géneros en la vivencia de la intensidad emocional. La cooperación suscita en las mujeres emociones positivas más intensas que en los hombres y también menor intensidad de emociones negativas y ambiguas. Estos resultados concuerdan con otros estudios en los que las chicas prefieren la cooperación

Movimento, Porto Alegre, v. 20, n. 2, p. 593-618, abr.jun. de 2014. 
antes que realizar otras prácticas (JOHNSON; ENGELHARD, 1992; KNIGHT; CHAO, 1989; MCKAY et al., 2000). Además las mujeres experimentan emociones más intensas en las prácticas de introyección motriz. Estas tareas ponen a prueba la capacidad para tomar consciencia de sus sensaciones, prestarse atención y poder discriminar el estado de cualquier parte u órgano del cuerpo. La lógica interna de estas prácticas pone en marcha una motricidad consciente dirigida hacia el autoconocimiento, el control corporal y el equilibrio psicosomático (LAGARDERA, 2007; LAGARDERA; LAVEGA, 2003; ROVIRA, 2010). Los resultados sugieren pensar que las mujeres muestran mayor sensibilidad por este tipo de prácticas de atención interoceptiva que los hombres (CONTI et al., 2001; MCKAY et al., 2000; ROVIRA, 2010).

Paralelamente el análisis cualitativo pone en evidencia que el género establece dos tendencias. Los chicos orientan mayoritariamente sus comentarios hacia variables de la lógica interna de los juegos mientras que el género femenino comparte estos argumentos con otros aspectos externos a las situaciones motrices propuestas. Las chicas atribuyen la vivencia de emociones positivas al placer que origina la ayuda mutua con otras personas al intercambiarse un objeto en un juego, realizar una danza cooperativa o desplazarse lentamente. Pero a diferencia de los chicos también subrayan otros aspectos asociados al contexto (lógica externa) como haber actuado o reído con una amiga o tener recuerdos agradables de una situación realizada en la infancia. Estos resultados ayudan a comprender mejor la socialización de los roles de género a partir de los valores y significados psicosociales asociados a las tareas motrices (BASSI; DELLE FAVE, 2012; CSIKSZENTMIHALYI; MASSIMINI, 1985; KNIGHT; CHAO, 1989). La tendencia prosocial manifestada históricamente por las mujeres se confirma también en el ámbito de las prácticas motrices.

La aparición de comentarios referidos a cuestiones externas al juego es mayor cuando los protagonistas tienen historial deportivo. La huella que deja la práctica competitiva habitual, muy alejada de las situaciones de cooperación sin competición, parece estar

Movimento, Porto Alegre, v. 20, n. 2, p. 593-618, abr.jun. de 2014. 
en la raíz de este comportamiento (CSIKSZENTMIHALYI; MASSIMINI, 1985; CONTI et al., 2001). La expresión, la introyección o los juegos sin competición introducen al alumnado en un escenario de desafíos motores donde no existe la comparación, ni la distinción de ganadores o perdedores. Esto podría explicar que para los alumnos con historial deportivo sea más difícil entregarse plenamente y fluir a través de la motivación intrínseca de este tipo de prácticas motrices (ENGESER; RHEINBERG; 2008; KELLER; BLESS, 2008) y que por tanto la vivencia emocional esté asociada a aspectos externos al juego: e.g., participar con un amigo (relación externa); recordar vivencias de la infancia (tiempo externo) o sorprenderse por un material nuevo que no conocía como el paracaídas (material externo).

Las narraciones femeninas hacen mucha más referencia a los rasgos de la lógica interna de la cooperación (interacción motriz, tiempo y uso del espacio) que los textos de los hombres. El género femenino, más próximo culturalmente a participar de relaciones motrices cooperativas, encuentra mayor bienestar socioemocional al intercambiar generosidad y empatía con los demás, tal y como apuntan otros estudios (CONTI et al., 2001; JOHNSON; ENGELHARD, 1992; KNIGHT; CHAO, 1989).

\section{Conclusiones}

Este trabajo contribuye a desvelar la relevancia de las prácticas motrices cooperativas en la promoción del bienestar socioemocional de los alumnos practicantes. Los hallazgos constatan una tendencia significativa en la relación entre las prácticas cooperativas y la vivencia de emociones positivas. Estos resultados confirman la naturaleza compleja y multidimensional de las emociones (BISQUERRA, 2003; LAZARUS, 1991).

El estudio de las prácticas motrices y las vivencias emocionales que se desencadenan con ellas exige atender a dos planos distintos; por una parte a la necesidad de identificar previamente los rasgos pertinentes de las situaciones motrices seleccionadas

Movimento, Porto Alegre, v. 20, n. 2, p. 593-618, abr.jun. de 2014. 
(lógica interna). Por otra, la vivencia emocional también depende del significado que tiene la situación experimentada según las expectativas de los protagonistas (ELIAS, 1987). La interpretación de la vivencia emocional se logra a partir de tener en cuenta y examinar estos dos niveles interconectados (PARLEBAS, 2001).

Los resultados aportan argumentos para reconocer el valor de la cooperación y de las prácticas motrices minoritarias aún hoy en EF, como son los juegos tradicionales, la expresión motriz y la introyección motriz, para educar el bienestar social y emocional (LAGARDERA, 2007; LAVEGA et al., 2011; TORRENTS et al., 2011). Además se confirma la necesidad de considerar la variable género ya que los efectos socioemocionales son desiguales en hombres y mujeres (JOHNSON; ENGELHARD, 1992) y el historial deportivo (CHALABAEV, et al., 2013).

El profesorado de EF que desee utilizar la cooperación motriz para generar experiencias de bienestar, dispone de un abanico extraordinario de posibilidades motrices para enriquecer las vivencias socioemocionales de su alumnado (LAGARDERA, 2007; LAVEGA, et al., 2011; LAVEGA et al., 2013). Por una parte las situaciones cooperativas de juegos tradicionales y de expresión motriz pueden estimular experiencias intensas de bienestar subjetivo y social al invitar al alumnado a solucionar retos compartidos, ya sea mediante la cooperación instrumental (en los juegos tradicionales) o a través de la interacción cooperativa y la relación referencial (en la expresión motriz). Por otra parte, el profesorado de EF puede promover experiencias positivas dirigidas a la autoexploración mediante prácticas motrices introyectivas, que posibiliten un apasionante viaje consciente por el interior de sus conductas motrices.

En definitiva, los resultados aportan razones de peso para afirmar que la cooperación motriz mediante los juegos tradicionales y la expresión motriz o a través de las prácticas motrices de introyección, constituye una familia de experiencias motrices necesaria en cualquier programa de intervención de EF que pretenda favorecer el bienestar socioemocional del alumnado.

Movimento, Porto Alegre, v. 20, n. 2, p. 593-618, abr./jun. de 2014. 
Efeito da cooperação motriz na vivência emocional positiva: perspectiva de gênero

Resumo: Investigou-se, desde uma perspectiva de gênero, a vivência emocional provocada pela prática de situações motrizes cooperativas. Participaram 309 estudantes de quatro universidades espanholas. Utilizou-se a escala validada de jogos e emoções (GES). Depois de cada tarefa, os alunos indicaram a intensidade experimentada nas treze emoções consideradas e comentaram brevemente as causas que originaram a emoção mais intensa. Utilizouse métodos mistos ao complementar a análise dos dados quantitativos com o estudo de comentários qualitativos. Confirmou-se a contribuição de situações motrizes de jogos tradicionais, expressão e introjeção para promover emoções positivas em mulheres e homens.

Palavras chave: Educação Física. Inteligência emocional. Identidade de Gênero. Relações interpessoais.

\section{Effect of motor cooperation on positive emotional: a} gender perspective

Abstract: We examined the relationship between gender and the experience of in the context of cooperative motor tasks. Participants were 309 students from four Spanish universities. Emotions were rated using a validated instrument (GES; Games and Emotions Scale). After each task, students were asked to rate the intensity of thirteen emotions, and to comment briefly on why they thought they had experienced the strongest emotion. A mixed methods approach was used, combining the analysis of quantitative data with an analysis of students' comments. The results confirmed the important contribution which traditional games, motor expression and the internalization of motor skills can make in terms of promoting positive emotional experiences in women and men.

Keywords: Physical Education. Emotional intelligence. Gender identity. Interpersonal reations.

\section{REFERENCIAS}

ANDRADE, Elena. et al. Modelo de medida del estado de ánimo subjetivo en deportistas adolescentes. Revista de Psicología del Deporte, Mallorca, v.20, n. 2, p.537-548, 2011.

BASSI, Marta; DELLE FAVE, Antonella. Optimal experience and self-detemination at school: Joining perspectives. Motivation and Emotion, Boston, v. 36, n.4, p.111, 2012.

Movimento, Porto Alegre, v. 20, n. 2, p. 593-618, abr.jun. de 2014. 
BERICAT, Eduardo. La integración de los métodos cuantitativo y cualitativo en la investigación social: Significado y Medida. Barcelona: Ariel, 1998.

BISQUERRA, Rafael. Educación emocional y competencias básicas para la vida. Revista de Investigación Educativa (RIE), Murcia, v.2, n. 1, p.7-43, 2003.

CHALABAEV, Aina. et al. The influence of sex stereotypes and gender roles on participation and performance in sport and exercise: Review and future directions. Psychology of Sport and Exercise, Atlanta, n.14, p.136-144, 2013.

COHEN, Jacob. A Coefficient of Agreement for Nominal Scales. Educational and Psychological Measurement, Londres, n.20, p.37-46, 1960.

CONTI, Regina; COLLINS, Mary Ann; PICARIELLO, Martha. Personality and Individual Differences, Londres, n.30, p.1273-1289, 2001.

CSIKSZENTMIHALYI, Mihaly; MASSIMINI, Fausto. On the psychological selection of bio-cultural information. New Ideas in Psychology, Bethlehem, n. 3, p. 115-138, 1985.

ELIAS, Norbert. On Human Beings and their Emotions: A Process-Sociological Essay. Theory, Culture \& Society, Londres, v. 4. n. 2/3, p. 339-361, 1987.

ENGESER, Stefa; RHEINBERG, Falko. Flow, performance and moderators of challenge-skill balance. Motivation and Emotion, Boston, n. 32, p. 158-172, 2008

ETXEBESTE, Joseba. À cloche-pied. Les jeux sportifs traditionnels et la socialisation des enfants basques. Sarrebruck: Editions Universitaires Europeennes, 2012.

FREDRICKSON, Barbara. What good are positive emotions? Review of General Psychology, Washington, n. 2, p. 300-319, 1998.

HEKTNER, Joel. Family, school, and community predictors of adolescent growth conductive experiences: Global and specific approaches. Applied Developmental Science, Londres, n.5, p. 172-183, 2001.

JOHNSON, Charlene; ENGELHARD, George. Gender, academic achievement, and preferences for cooperative, competitive, and individualistic learning. The Journal of Psychology, Londres, n.126, p.385-392, 1992.

KELLER, Johannes; BLESS, Herbert. Flow and regulatory compatibility: An experimental approach to the flow model of intrinsic motivation. Personality and Social Psychology Bulletin, v.34, n. 196-209, 2008.

KNIGHT, George; CHAO, Chia-Chen. Gender differences in the cooperative, competitive, and individualistic social values of children. Motivation and Emotion, Boston, v. 13, n. 125-141, 1989.

LAGARDERA, Francisco. Ejercicio físico y bienestar: Las prácticas motrices introyectivas en el INEFC de la universidad de Lleida. Lleida: Universidad de Lleida, 2007. 
LAGARDERA, Francisco; LAVEGA, Pere. Introducción a la praxiología motriz. Barcelona: Paidotribo, 2003.

LAVEGA, Pere. La investigación en los juegos tradicionales y en los juegos cooperativos. In: NAVARRO, Vicente; TRIGUEROS, Carmen (org.). Investigación y juego motor en España. Lleida: Universidad de Lleida, 2009. p. 77-116.

LAVEGA, Pere. et. al. Understanding emotions through games: Helping trainee teachers to make decisions. Electronic Journal of Research in Educational Psychology, Almería, v.9, n. 2, p. 617-640, 2011.

LAVEGA, Pere; MARCH, Jaume; FILELLA, Gemma. Juegos deportivos y emociones. Propiedades psicométricas de la escala GES para ser aplicada en la Educación Física y el Deporte. Revista de Investigación Educativa, Murcia, v. 31, n.1, p.151-165, 2013.

LAZARUS, Richard. Emotion and adaptation. New York: Oxford University Press, 1991.

MARIN, Carolina E. et al. Jogos tradicionais no Estado do Rio Grande do Sul: manifestação pulsante e silenciada. Movimento, Porto Alegre, v. 18, n. 3, p. 73-94, 2012.

MASSIMINI Fausto; DELLE FAVE, Antonella. Individual development in a biocultural perspective. American Psychologist, Washington, n.55, p.24-33, 2000.

MATEU, Mercè. Observación y análisis de la expresión motriz escénica. Estudio de la lógica interna de los espectáculos artísticos profesionales: Cirque du Soleil (1986-2005). Tesis doctoral. INEFC. Barcelona: Universidad de Barcelona. 2010.

MCCAUGHTRY, Nate .The emotional dimensions of a teacher's pedagogical content knowledge: Influences on content, curriculum, and pedagogy. Journal of Teaching in Physical Education, Champaign, v. 23, n. 1, p.30-47, 2004.

MCKAY, Jim; MESSNER, Michael; SABO, Donald (Org.). Masculinities, gender relations, and sport. London: Sage, 2000.

PARLEBAS, Pierre. Juegos, Deporte y Sociedad: Léxico de Praxiología Motriz. Barcelona: Paidotribo, 2001.

PEKRUN, Reinhard; ELLIOT, Andrew; MAIER, Markus. Achievement goals and achievement emotions: Testing a model of their joint relations with academic performance. Journal of Educational Psychology, Washington, n.101, p. 115135, 2009.

ROVIRA, Glòria. Prácticas motrices introyectivas: una vía práctica para desarrollo de competencias socio-personales. Acción Motriz, Las Palmas de Gran Canaria, n.5, p. 12-19, 2010.

RYAN, Richard; DECI, Edward. On happiness and human potentials: a review of research on hedonic and eudaimonic well-being. Annual Review of Psychology, Palo Alto, n.52, p.141-166, 2001.

Movimento, Porto Alegre, v. 20, n. 2, p. 593-618, abr.jun. de 2014. 
TORRENTS, Carlota, et al. Posibilidades de las tareas de expresión corporal para suscitar emociones en el alumnado. Revista de Psicología del Deporte, Mallorca, v. 20, n.2, p. $401-412,2011$.

Fonte de financiamento: Ministerio de Economía y Competitividad, Secretaría de Estado de Investigación, Desarrollo e Innovación. Proyecto I+D+i. Referencia. DEP2010-21626-C03-01.

Endereço para correspondência:

Pere Lavega

INEFC (Universidad de Lleida)

Partida Caparrella s/n

25192 Lleida (España)

Recebido em: 29.03.2013

Aprovado em: 02.03.2014

Movimento, Porto Alegre, v. 20, n. 2, p. 593-618, abr.jun. de 2014. 